\title{
The Effects of a Recovery-Focused Program for Stress Management in Women
}

\section{-An Exploratory Study}

\author{
Jan Lisspers ${ }^{1,2}$, Niclas Almén1, Örjan Sundin ${ }^{1}$ \\ ${ }^{1}$ Department of Psychology, Mid Sweden University Campus Östersund, Östersund, Sweden \\ ${ }^{2}$ Swedish Winter Sports \& Research Center, Mid Sweden University Campus Östersund, Östersund, Sweden \\ Email: Jan.Lisspers@miun.se
}

Received 3 October 2014; revised 16 November 2014; accepted 2 December 2014

Copyright (C) 2014 by authors and Scientific Research Publishing Inc.

This work is licensed under the Creative Commons Attribution International License (CC BY). http://creativecommons.org/licenses/by/4.0/

(c) (i) Open Access

\begin{abstract}
Current research indicates that stress problems primarily could be conceptualized as deficiencies in recovery and recuperation between stress periods. Accordingly, interventions should put more emphasis on this aspect. A group based intervention program focusing exclusively on recovery behavior in everyday life was evaluated in this quasi-experimental, waiting-list control group study, where the control group was also treated in a second phase. Thirty-two self-referred female subjects, considering themselves in need of treatment for stress related health problems, were available for analyzes. Fifteen of these constituted the first phase treatment group (INT), while the remaining 17 subjects were placed on waiting list (WLC). Adding a few late applicants leaved 20 subjects later treated in the second intervention phase. Significant and clinically meaningful positive effects emerged in the INT - compared to the WLC-group on recovery behaviors, stress-and recovery experiences, as well as on burn-out symptoms, worry, anxiety and depression. Secondary analyzes of all treated subjects indicated that the positive change the primary clinical endpoint was predicted by the increase in frequency of recovery behaviors and by the decrease in the worry level. Thus, the present intervention model merits further research with more rigorous experimental design as well as with follow-up assessments.
\end{abstract}

\section{Keywords}

Stress, Recovery, Stress Management, Intervention, Cognitive Behavior Therapy

\section{Introduction}

Stress-related and other mental problems have become increasingly common causes of long-term illness. To- 
gether with so called "lifestyle diseases", in which stress responses also have a significant impact, these conditions together account for a large majority of the total number of people on sick leave (and thus the cost of sick leave payments) in Sweden [1]. The knowledge of how these stress-related problems should be prevented, treated and rehabilitated is today unfortunately_and somewhat surprisingly-still deficient. Consequently, much more theoretical, developmental and evaluative work of intervention models and formats seems to be needed.

Most theories about stress postulate that it is the prolonged, more or less continuous tension and physiological arousal that is harmful, possibly resulting in bodily injury and morbidity. This perspective, already evident in the earliest theoretical stress analyzes by for example [2], have however largely been neglected in contemporary research. McEwen's theory of “allostatic load” [3], as well as Ursin and Eriksen's “Cognitive Activation Theory” [4] [5] focusing the long-lasting aspects of the stressors and the stress reactions, are in this respect interesting and important exceptions.

That long-term stress reaction occurrence has primarily been attributed to highly reactive individuals being exposed to prolonged and/or high frequency (external) stressors. This analysis does however not seem to be sufficient for explaining how stress responses in daily life can cause human illness [6]-[8]. Both anticipatory reactions before exposure to an actual or potential stressor, as well as deficiencies in the recovery after exposure are necessary parameters for an understanding of how stress reactions in daily life can be sufficiently prolonged to cause adverse effects [6] [9]. Relatively recent theoretical analyzes and empirical studies also emphasize the important role of perseverative, i.e. persistent, repeated and thus extended cognitions and emotions ("perseverative cognition”) as for example anxious ruminations and worry [9]-[12].

Recovery can be conceptualized as a process of psychophysiological deactivation after effort expenditure, which is the opposite of the psychophysiological activation that occurs during strain or stress [13]. Recovery occurs in the absence of demands, which allows the arousal level to return to baseline, depleted resources to be restored, fatigue reduced, and existing or future demands be dealt with more efficiently [14]. Thus recovery, especially repeated recovery, would be instrumental in counteracting the harmful prolongation of everyday life stress reactions [13].

In a series of experimental studies Christenfeld and co-workers showed that emotional stressors resulted in slower recovery than emotionally neutral ones [15], distracting activity or moderate physical activity after an emotional stressor resulted in faster recovery than merely passive non-activity [16] [17], and social support as well as listening to some (but not all) types of music facilitated recovery [18] [19]. At the very least, findings such as these indicate that recovery surely is not a simple straightforward or static process, and that there is still much left to be investigated, for example how recovery can be facilitated in everyday life.

Being central to recovery and recuperation, sleep can be considered as the ultimate form of deactivation. Prolonged stress reactions and lack of recovery during the day increases the risk of disturbed sleep [20], while on the other hand sleep problems in themselves represent a serious disturbance in recovery subsequently increasing the stress load [21] and the risk for development of fatigue, exhaustion, and "burnout” [22]. For example, sleep and different aspects of recovery during leisure time appear impaired or insufficient in burnout subjects compared to controls [23], and the recovery from burnout seems to be related to corresponding positive changes in sleep physiology [24]. Furthermore, Akerstedt and co-workers [25] showed that self-reported bedtime stress and worries resulted in impaired sleep quality, and Brosschot, Van Dijk and Thayer [26] found that the degree of worry during day-time was related to physiological indicators of impaired recovery during the subsequent night, even without self-reported sleep disturbances-adding to the arguments for the importance of recovery during everyday life. This might be especially salient for women.

Recent official statistics in Sweden show that almost twice as many women (14\%) than men report ailments due to stress or other psychological encumberments related to work. The so-called "work-family interface" seem to be related to stress levels experienced and especially to recovery possibilities out of work [27]. This is probably relevant for example concerning the "unpaid work" (household chores, child caring, and so on), where the gender differences have slowly decreased in Sweden, in that women on average have reduced the time spent on unpaid work since the beginning of the 90 -ies by one hour (to 3.5 hours), while men have increased their time to 2.5 hours-by 8 minutes [28]. The idea that such circumstances is linked to decreased possibility for recovery and recuperation for women fits well with the classical studies by Frankenhaeuser, Lundberg and co-workers who showed that women's stress levels (catecholamine levels) remained elevated long after work, while men rapidly returned to baseline levels at the end of the workday [29] [30]. Overall it seems that “...full-time em- 
ployed women are often exposed to more stress at work and have fewer opportunities for rest and recovery off work compared with men” [31], conditions that might co-occur with or lead to increased anxiety and depression symptoms [32].

In line with the central importance, we have placed on recovery as a way of counteracting the prolongation of stress reactions in everyday life as outlined above, the general intervention approach adopted here could be better characterized more as "recovery management" rather than "stress management". The focus is not primarily to help clients with ways to observe, analyze, avoid, cope with or resolve real life stressors per se, and not to teach them how to cope with or reduce the actual and short term cognitive, emotional and/or physiological stress reactions. Instead we endeavor to help increase the frequency and efficiency of recovery and recuperation behavior after and in-between stress situations in everyday life. If this turns out to be successful, it would obviously reduce the harmful long duration of stress reactions (which is supposed to be the main detrimental aspect of stress reactions) - and thus reduce also the negative effects of stress on health. Therefore, the aim of the present, first exploratory study of this model, was to analyze what results can be achieved in treatment of stress related problems with an intervention method strongly and narrowly focused on increasing the frequency of recovery behavior in everyday life, and on improving the ability of deactivation and recovery in such occasions. The short-term effects of the intervention will be compared to a no-treatment waiting-list group. Furthermore, we will in secondary analyzes explore to what degree the primary focus of the intervention model, i.e. the changes in frequency of recovery behavior, were in fact, as hypothesized, related to the clinical effects achieved after treatment.

\section{Method}

\subsection{Procedure and Design}

Recruitment of participants to the study was carried out as part of a joint project between the local city council of Ostersund and the Winter Sports \& Research Center (NVC) at Mid Sweden University, where a total of fifty positions in our program were offered during two different sequential intervention phases. Due to practical reasons in this particular cooperation project, a randomized allocation of subjects to different experimental conditions was unfortunately not possible. Thus, staff members were recruited in and by different departments of the organization and applied for participation themselves in either of the two phases and in one of three different treatment groups (see below) in each phase of their own choice. To start with, applications were accepted for the first phase only, and once the first phase had filled up, applications for the second phase were accepted.

As part of the PRE-assessment procedure (see below), all enrolled participants (in both intervention phases) were given an explicit description of the planned evaluation study and were asked to give their (signed) informed consent of participation in the study. It was explicitly stated that participation in the study per se was not a requirement for participation in the intervention program. Since very few males applied for participation, only female subjects will be analyzed in the present report ${ }^{1}$.

Those accepting participation in the evaluation study (and thus included in the present analyzes) as well took part in somewhat more elaborate assessments than those declining, with a few more questionnaires to answer and more importantly, those recruited for the second intervention phase, also took part in additional assessments during the waiting period until the start of their intervention.

A total of 21 female employees originally applied and were accepted for participation in the first treatment wave. Out of these, four declined participation in the study and two dropped out during treatment, leaving 15 subjects for analysis in the intervention group. For the second treatment wave 23 employees (out of the 25 positions available) applied in time to be offered participation. Nineteen out of these accepted participation in the study, and 17 completed the waiting-list period assessments, thus constituting the waiting-list comparison group. Two of the former waiting-list group subjects subsequently declined participation in the intervention, and five late applications were accepted leaving a total of 20 participants in the second wave intervention. This leaves a total of 35 subjects who completed the intervention and the pre-post assessments in either the first or the second wave.

${ }^{1}$ Out of a total of forty-three applicants, only eight were males and all of these applied to the first treatment phase precluding any meaningful comparative analyses to no-treatment WLC-controls.. 


\subsection{Intervention Program}

The intervention consisted of six 3-hour sessions scheduled over a period ten weeks, one session a week during the first three weeks, with successively greater intervals between sessions as time progressed (the last two sessions were scheduled three weeks apart). The formal intervention program described below was conducted during the first two hours of each session at the Mid Sweden University Psychological Clinic. In addition, the last hour provided the participants with the opportunity to try out a variety of different, for many participants new, examples of potentially useful recovery behaviors such as Qi-gong, massage, relaxation training (in a special "recovery friendly" environment), and "Nordic walking" under the guidance of personnel at the Swedish Winter Sports \& Research Center, NVC on Campus.

Sessions were conducted in groups of 6 - 9 participants. One of the groups during each phase was run by an experienced group leader (the second author) who is a licensed psychologist, as well as a licensed psychotherapist, trained in Cognitive Behavior Therapy and with extensive clinical experience in this area. During each phase, each of the two remaining groups were led by two clinical psychology Master of Science students ${ }^{2}$, who at the time were enrolled in the latter part of their clinical training. These students had attended a special course in stress management intervention techniques, and they had also been group leaders in an earlier pilot trial using the present program. During the whole intervention period they were provided tutorial supervision on a regular basis by an experienced clinical psychologist (the second author).

The main, and explicit, goal of the program was to promote behavior change in the participants' daily life in three main areas: a) to be observant on and sensitive to tension and arousal, as well as early signs of the negative stress-, distress- and fatigue/exhaustion effects of either of these; b) to frequently use different behaviors that have proved to provide opportunity for achieving recovery and recuperation effects; and c) to use tension release and relaxation skills ("Applied Tension Release, ATR" [33] [34] in such recovery situations to increase the efficiency of the deactivation and recuperation processes.

In order to promote such behavior change the intervention comprised the following general components:

- Psycho-education about stress and especially about the rationale for recovery behavior.

- Daily drills of different specific "recovery behaviors" in every-day life, at work and out of work (such as for example "Eat meals really slowly and calmly", "Take a slow walk and focus on walking in a relaxed and slow manner", "In a "waiting situation", try to calm down and relax").

- Relaxation training in the form of "Applied Tension Release (ATR)".

- Encouragement and assignments to use different individually tailored and/or preferred new or regular recovery behaviors in everyday life.

- Social reinforcement, and focus on experiences of positive recovery effects, of behavior change.

- Problem solving to facilitate change in recovery behavior.

- Self-monitoring (behavioral diaries) of recovery behaviors drills and relaxation training.

- Self-monitoring (behavioral diaries) of recovery behaviors and usage of relaxation skills.

- Review and discussions of each participant's PRE-POST results of some relevant variables.

\subsection{Assessment}

Assessments were performed through the use of online questionnaires, where the subjects were given instructions and provided with a personal user id and password through e-mail at each assessment phase. For different reasons, a few of the subjects had trouble connecting to the online questionnaire system, and they were therefore sent corresponding questionnaires on paper through postal mail. The PRE-assessments were baseline assessment done before the start of each intervention phase, and the POST-assessments were done just before the very last group session.

The primary behavioral change endpoints chosen for this study were the self-reported frequency of recovery behaviors in every-day life, and were assessed with two different questionnaires developed especially for this project. The "Recovery Behavior Scale (RBS)" was developed especially for the present project to allow for subjects to specify which specific behaviors they normally perform in order to help recover and recuperate after stress and/or any kind of wearying activity of everyday life. The scale comprises twelve examples of specific behaviors judged to be commonly used such as: "I take a coffee break", "I sit or lie down and rest for a while",

${ }^{2}$ Sofia Djusberg, Johan Ekström, Johan Landin, and Amanda Simonsson. 
"I take a good break from doing something tiring”, "I use some formal method of relaxation, meditation, yoga or the like", and "I take a slow walk". These items were to be judged according to how frequently the behavior is used on a five-point Likert scale from "Never" (0) to "At least once a day" (4), and the mean was calculated and used as an overall frequency score. The "Recovery at Work Questionnaire (RWQ)", also developed especially for this project, covers the frequency of pauses during working hours. The six items include for example "How often do you take a break from what you are working with?", "How often do you choose a completely different work task in order to get a break and try to wind down?", and "How often do you take a coffee break (for at least 10 minutes)?”. A five-point Likert scale from "Never" (0) to "Several times a day" (4) was used, and the mean was calculated and used as an overall frequency score.

A crude combined index named “Combined Recovery Behavior Frequency Index (CRBFx)" (0 - 4) was calculated as the mean of the overall scores of these two scales.

Among the other stress-related behavioral variables analyzed are type A-behavior which was assessed using a 9-item version of “The Everyday Life Stress scale (ELS)" [35] [36]. This questionnaire consists of statements referring to stress behavior in everyday life, focusing mainly time pressure-urgency-impatience and easily aroused irritation or hostility, and items are rated for frequency of experience on a four-point (0 - 3; “almost never”-“almost always”) scale. Over commitment to work (OCW) was assessed using the 6-item (scale 1 - 4) subscale of the "ERI questionnaire" [37]-[39], and worry with the "ultra-brief" three-item version [40] of the original “Penn State Worry Questionnaire (PSWQ)” [41].

The two primary clinical effects endpoints of the study were a) self-reported feelings of general stress; and b) self-reported feelings of recovery and recuperation. The experience of general stress was assessed through the 10 item version of "Perceived Stress Scale (PSS)", [42] designed to examine the extent to which situations in an individual's life are perceived as unpredictable, uncontrollable and overloading. The answers were given on a five-point Likert scale ranging from 0 ("Never") to 4 ("Very often”). Feelings of recovery and recuperation were assessed using the "Recovery Scale (RCS)" [43] [44]. This eight-item scale includes items assessing recovery, recuperation, and fatigue as related to work at different times of the day (in the morning, after work, and after the weekend). The answers were rated on a five-point Likert scale from 1 ("Never"), to 5 ("Very often"), and the mean was calculated were high scores indicate that individuals have difficulties recovering.

As for the CRBFx above, a crude combined index named "Combined Clinical Stress and Recovery Index $(C C S R x)$ " (0 - 4) was calculated as the mean of the overall scores of these two scales, after transforming the item-scores on the RCS-scale to 0 - 4.

The "Shirom-Melamed Burnout Questionnaire (SMBQ)", consisting of 22 items, graded 1 - 7, was used to measure burnout symptoms by subscales of emotional and physical fatigue/exhaustion, cognitive fatigue/deficits, apathy/indifference and tension [45]-[47]. The average score, thus ranging from 1 - 7, for the overall index (SMBQ-global) was calculated. “Hospital Anxiety and Depression Scale (HAD)” comprising two subscales, was used to measure anxiety (HAD-A) and depression (HAD-D) respectively. Each subscale consists of seven items which are graded from 0 to 3, yielding sum-scores of 0 - 21 [48] [49]. Finally, sleep and sleep problems were measured by means of the 3-item "sleep quality index" (scored 1-5) of the "Karolinska Sleep Questionnaire (KSQ)", [50] [51].

\subsection{Statistical Methods}

Analyzes of the effects of the intervention were performed in two steps. The mean changes from PRE- to POSTassessments were analyzed for the first intervention phase, i.e. the intervention (INT) group compared to the waiting-list no-treatment control group (WLC). In a second step, the change during the treatment phase for the earlier waiting-list group was compared to the original treatment group's results. Analyzes were performed using repeated measures ANOVA, with a critical alpha-level of .05 adopted in all analyzes. Effect sizes were computed using Cohen's $d$ score [52] for pre-post control group designs [53] and interpreted as small ( $\geq 0.2$ ), medium $(\geq 0.5)$ or large $(\geq 0.8)$ (Cohen, 1992). For the prediction analysis, linear multiple regression analysis was performed on crude change-scores (PRE-POST) computed for the separate behavioral variables and for the main clinical outcome index (CCSRx).

The study protocol was approved by the Regional Ethical Review Board in Umeå, Sweden (Dnr 2011-277-31 O). Statistical analyzes were performed using the STATISTICA data analysis software system, version 10 (StatSoft, Inc., 2011) and IBM SPSS Statistics, version 21 (SPSS IBM, 2012). 


\section{Results}

\subsection{Effects of the Intervention Compared to Waiting List No-Treatment Control}

There were no significant differences between the groups on any of the variables at the PRE-assessments.

As Figure 1 shows, there was a significant group*phase interaction in the repeated measures ANOVA yielding a medium effect size (Table 1) for the primary behavioral change endpoints (Combined Recovery Behavior Frequency Index; CRBFx), where the mean score for the INT-group, but not for the WLC-group, increased significantly during the intervention phase. At the end of the intervention, the mean frequency score for the intervention group was significantly higher (better) than the control group. Table 1, which summarizes the results of the study, shows that the same picture emerges in analyzes of the two separate recovery behavior frequency variables (in and out of work respectively), with significant interaction effects, medium effect sizes, as well as significant within-group mean changes for the INT- but not the WLC-group, also resulting in significant between-group differences at the end of the intervention phase favoring the intervention. All the other three analyzed behavioral factors also showed significant interaction effects with large (ELS) or medium (OCW, PSWQ) effect sizes, and significant within-group changes for the INT-group but not for the WLC-group (Table 1). Only the worry-variable (PSWQ) however reached significant between-group differences at the end of treatment.

Figure 2 illustrates the results of the group*phase repeated measures ANOVA of the primary clinical effects endpoints index (CCSRx). There emerged a significant interaction effect, with a large effect size (Table 1). The INT-group, contrary to the WLC-group, showed a significantly reduced mean level in the ratings of stress- and recovery problems during the treatment phase, and at the end of intervention the between-group difference was significantly in favor of the intervention group.

Both measures of the perceived stress (PSS) and the feelings of recovery and recuperation (RCS), which the CCSRx-index is based on, separately showed comparable favorable results for the intervention group, and yielding large and medium effects sizes respectively (Table 1).

Regarding the other clinical effects analyzed here (see Table 1) both burn-out symptoms (SMBQ; medium es.), anxiety (HAD-A; medium es.) and depression (HAD-D; large es.) showed positive effects in favor of the INT-group (significant group*phase interaction effects, and significant within-group change in expected direction only for the intervention group). Changes in sleep problems rating (KSQ) were rather small, and though there is some tendency for arithmetically larger mean change for the INT-group, no significant effects were found.

\subsection{Comparing Results of the Two Intervention Phases}

There were no significant differences on any of the variables between the original intervention group (INT) and the earlier waiting-list control group (WLC) at the start of their respective intervention phase.

No significant (group*phase) interaction effects emerged, indicating that there were no differences in intervention effects between the two waves. The overall main effect of phase (PRE-POST) was highly significant both for the primary behavioral change endpoints index $(\mathrm{CRBFx}: \mathrm{F}(1)=55.73$; $\mathrm{p}<0.0000001)$ and the primary clinical effects endpoints index (CCSRx:F(1) = 37.41; $\mathrm{p} \leq 0.00001)$.

\subsection{Relation between Behavioral Change and Clinical Effects}

In order to analyze which behavioral changes were related to the achieved clinical effects of the intervention, the PRE-POST change scores in self-reported frequency of recovery behaviors out-of work (RBS) and at work (RWQ), as well as in the self-ratings of Type-A behavior (ELS), overcomittment to work (OCW), and worry (PSWQ) were entered as predictors in a regression analysis with change in the Combined Clinical Stress and Recovery index (CCSRx) as the dependent factor.

The prediction model was significant $(F(5,29)=4.905, \mathrm{p}=0.002)$, and accounted for approximately $37 \%$ of the variance in CCSRx ( $2=0.458$, Adjusted R2 $=0.365)$. The change in frequency of recovery behaviors at work (RWQ) received the strongest weight in the model (beta $=-0.473, t(29)=3.056, p=0.005$ ), and the only other variable significantly associated with change in the outcome variable was change in worry (PSWQ; beta = 0.337, $\mathrm{t}(29)=2.328, \mathrm{p}=0.027)$.

\section{Discussion}

The main results of the present first study of the new intervention model support the overall hypothesis that fo- 
cusing intervention efforts narrowly on strengthening the women's recovery behavior is a productive way of treating stress related problems. Firstly, the achieved results indicate that it is in fact possible to help participants to increase the frequency of recovery attempts in everyday life without specifically and explicitly addressing the

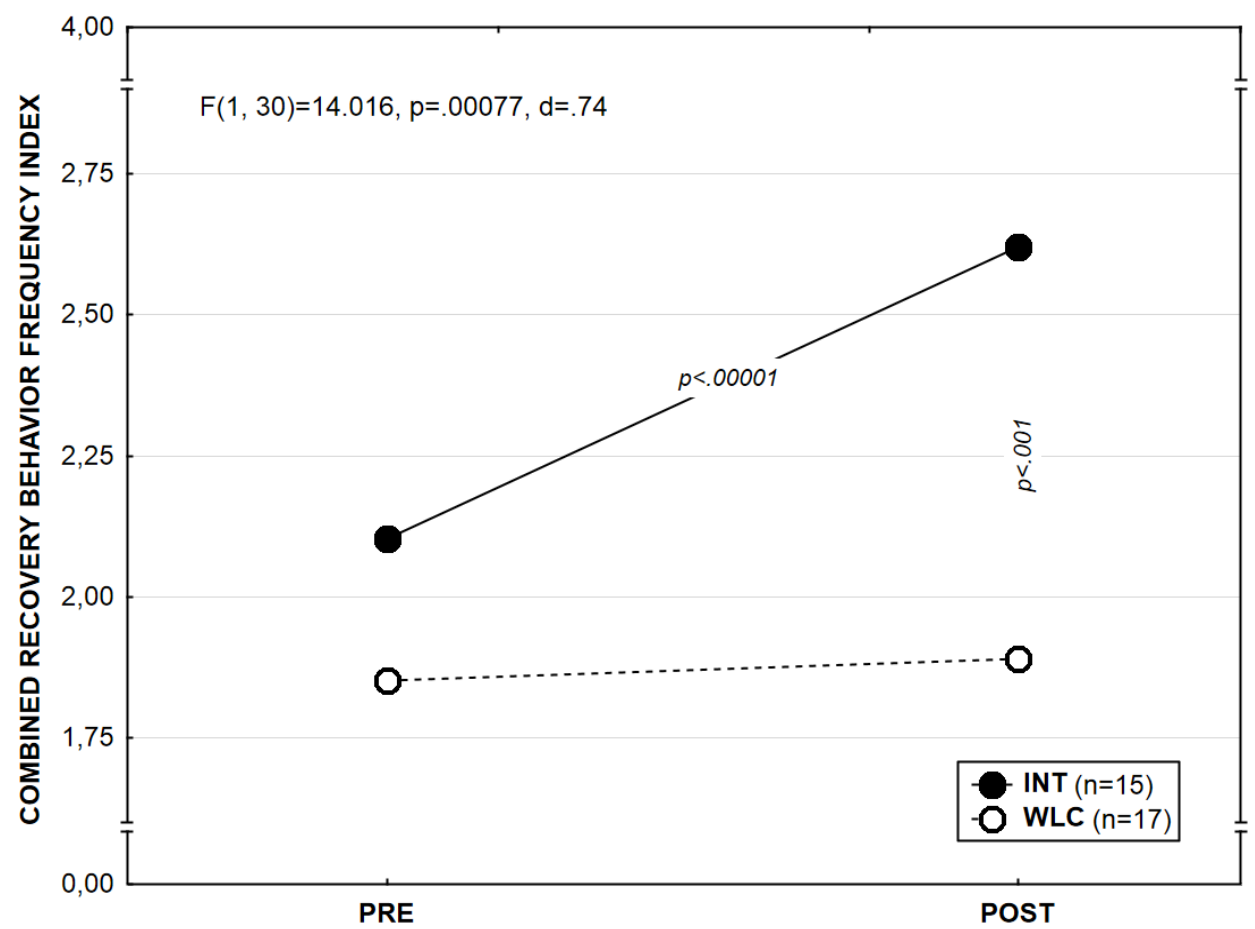

Figure 1. Self-reported frequency of recovery behaviors (CRBFx-index) before (PRE) and after (POST) the treatment phase for the intervention (INT) and the waiting-list group (WLC).

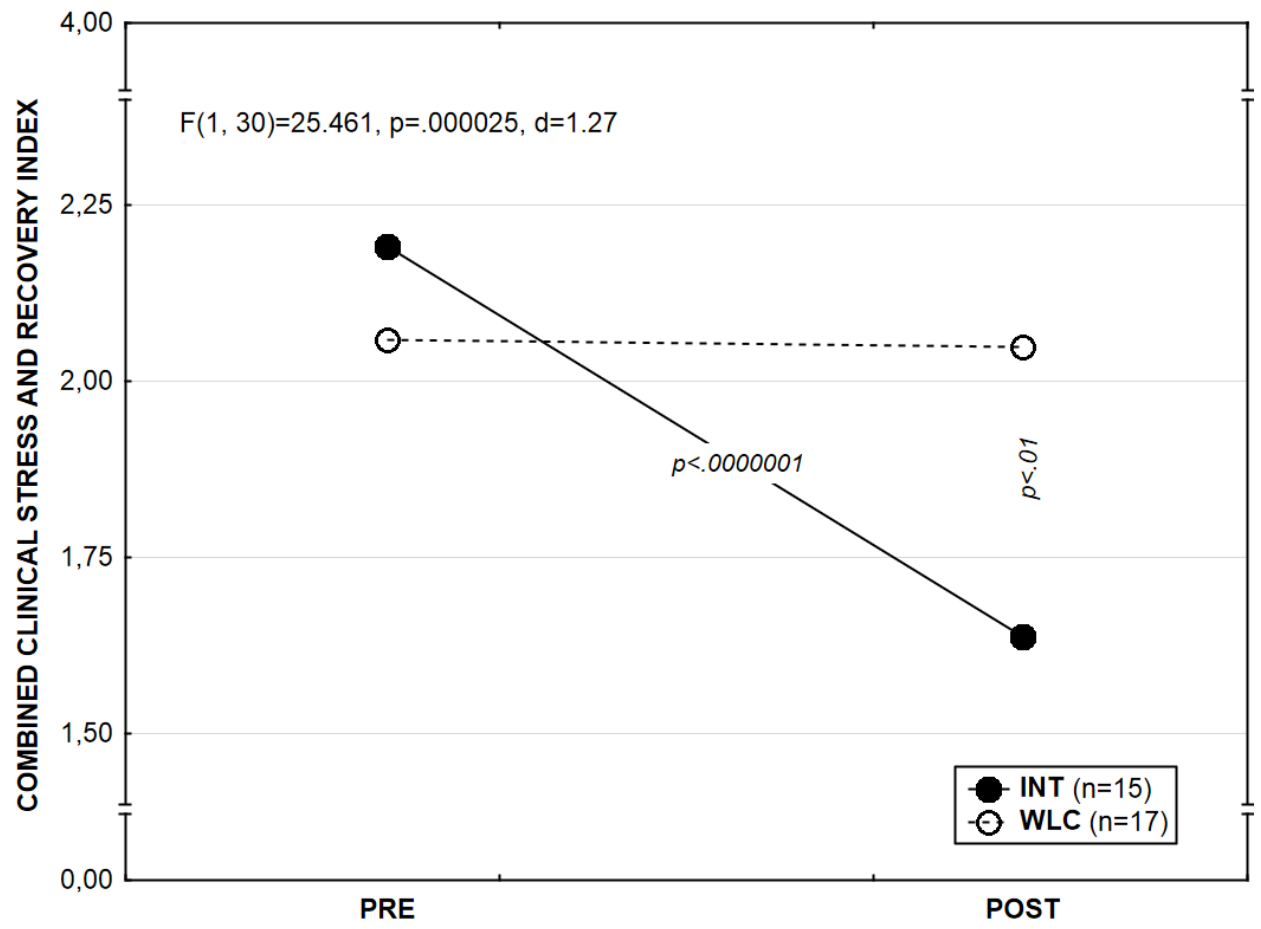

Figure 2. Self-rated levels of stress- and recovery problems (CCSRx-index) before (PRE) and after (POST) the treatment phase for the intervention (INT) and the waiting-list group (WLC). 
Table 1. Summary of results from the Repeated Measures ANOVA analysis of behavioral and clinical factors.

\begin{tabular}{|c|c|c|c|c|c|c|c|c|}
\hline \multirow[t]{2}{*}{ Variables } & \multirow[t]{2}{*}{ Scale } & \multicolumn{2}{|c|}{ Intervention } & \multicolumn{2}{|c|}{ Control } & \multirow{2}{*}{$\begin{array}{c}\mathrm{F} \text { (int.) } \\
\mathrm{p}<\end{array}$} & \multicolumn{2}{|c|}{ Eff. size } \\
\hline & & Pre & Post & Pre & Post & & d & Level \\
\hline \multicolumn{9}{|l|}{ Primary behavioral change end-points: } \\
\hline FRQ of recovery behaviors, out of work & RBS & $2.17^{\mathrm{a}}$ & $2.83^{\mathrm{b}}$ & 1.83 & $1.96^{\mathrm{a}}$ & 0.025 & 0.67 & Medium \\
\hline FRQ of recovery behaviors, at work & RWQ & $2.03^{\mathrm{a}}$ & $2.41^{\mathrm{b}}$ & 1.87 & $1.82^{\mathrm{a}}$ & 0.01 & 0.60 & Medium \\
\hline Combined Recovery Behavior Frequency & CRBFx & $2.10^{\mathrm{a}}$ & $2.62^{\mathrm{b}}$ & 1.85 & $1.89^{\mathrm{a}}$ & 0.001 & 0.74 & Medium \\
\hline \multicolumn{9}{|l|}{ Other stress-related behavioral factors: } \\
\hline Type-A behavior & ELS & $1.82^{\mathrm{a}}$ & $1.36^{\mathrm{b}}$ & 1.48 & 1.56 & 0.0005 & 0.85 & Large \\
\hline Overcomittment to work & OCW & $2.68^{\mathrm{a}}$ & $2.41^{\mathrm{b}}$ & 2.71 & 2.80 & 0.05 & 0.52 & Medium \\
\hline Worry & PSWQ & $2.80^{\mathrm{a}}$ & $2.16^{\mathrm{b}}$ & 2.86 & $2.92^{\mathrm{a}}$ & 0.025 & 0.67 & Medium \\
\hline \multicolumn{9}{|l|}{ Primary clinical effects end-points: } \\
\hline Perceived general stress & PSS & $1.93^{\mathrm{a}}$ & $1.48^{\mathrm{b}}$ & 1.82 & $1.84^{\mathrm{a}}$ & 0.025 & 1.17 & Large \\
\hline Feelings of recovery and recuperation & RCS & $3.45^{\mathrm{a}}$ & $2.79^{\mathrm{b}}$ & 3.29 & $3.26^{\mathrm{a}}$ & 0.00025 & 0.89 & Large \\
\hline Combined Clinical Stress and Recovery & CCSRx & $2.19^{\mathrm{a}}$ & $1.64^{\mathrm{b}}$ & 2.06 & $2.05^{\mathrm{a}}$ & 0.000025 & 1.27 & Large \\
\hline \multicolumn{9}{|l|}{ Other stress-related clinical effects: } \\
\hline Burn-out symptoms & SMBQ & $4.48^{\mathrm{a}}$ & $3.60^{\mathrm{b}}$ & 3.94 & 4.01 & 0.0025 & 0.54 & Medium \\
\hline Anxiety & HAD-A & $9.20^{\mathrm{a}}$ & $5.46^{\mathrm{b}}$ & 8.65 & 8.00 & 0.005 & 0.60 & Medium \\
\hline Depression & HAD-D & $7.47^{\mathrm{a}}$ & $3.47^{\mathrm{b}}$ & 5.64 & 5.00 & 0.001 & 0.89 & Large \\
\hline Sleep problems & KSQ & 2.44 & 1.82 & 2.78 & 2.57 & ns. & 0.37 & Small \\
\hline
\end{tabular}

Intervention $n=15$; Control $n=17$. Means with different exponents are significantly different $(p<0.05)$.

actual stressors or stress exposures per se. Secondly, the increase coincided during the treatment phase with significant and clinically meaningful reductions in self-reported stress experiences, and in different stress-related factors such as anxiety, depression, and burnout/exhaustion, indicating that such specific behavior changes are in fact of functional importance to stress-related and other clinical problems.

The fact that change in frequency of recovery behaviors turned out to be the strongest predictor of clinical effects also supports the above tentative conclusion. Of the other factors included in the regression analysis, only the change in worry levels was significantly related to the clinical effects. This obviously fit well with the perspective put forward by Brosschot and others in which an important causal role is given to perseverative cognitions and emotions, i.e. primarily worrying, in health adverse effects of stress [9] [54] [55]. Research shows that women report more worry than men [56]-[59] which might indicate that such effects of worry on stress reactions also might be crucial for women. Taking into account also available data indicating clear gender differences in specific aspects of the worry process itself [59] [60], the causal importance of worry to stress reactions and how to most effectively target worry in treatment of stress problems seems important areas for future research.

The strength and the generalizability of any far-reaching conclusions are of course hampered by the apparent limitations in the present study design, especially considering the selection of the study sample, the non-random allocation of subjects to the experimental groups, as well as the usage of only self-report measures, and the absence of any follow-up assessments. However, the almost identical positive effects achieved after treatment in the earlier waiting-list control group (from its stable level during the waiting control period), lend support to the conclusion that the observed positive effects are related to-and a consequence of - the intervention activities. In an earlier exploratory pilot-study [61] we aimed at evaluating the effects of this treatment model in individual format using a multiple baseline (across three subjects) single subject experimental design [62] [63]. The results of that study indicated, with at least moderate experimental control, a casual (i.e. temporal) relation between the start of the active intervention and the change from baseline in continuous self-recordings of stress reactions. 
Also, follow-up assessments of relevant stress related clinical questionnaire variables showed excellent 12months' maintenance of post-treatment achieved changes for all three subjects. Taking this into account as well, we propose that the present results are positive as to the possibility of replication of the effects of the intervention. However, future studies with more rigorous experimental design as well as with follow-up assessments are obviously needed.

Most variables analyzed here showed comparable statistically significant and clinically meaningful positive changes with large effect sizes for the intervention group compared to the control group. The negative exception is sleep problems, measured with the sleep quality index of the Karolinska Sleep Questionnaire (KSQ). Since sleep-and thus the existence of sleep problems-is such an important factor in our daily recovery, we are somewhat concerned about the absence of positive intervention effects. The sensitivity to change of 3-item index of the KSQ is uncertain, and since there are no formal norms for the scale we cannot determine the proportion of subjects who in fact suffered from any sleep problems at the beginning of treatment. On the other hand, the intervention program did not include any measures specifically addressing sleep problems since we assumed the general recovery behavior aspects of the program would have an effect on sleep disorders, if any. Further analyzes of this matter is clearly needed, both in regards to the available data from the present study and in more elaborate future studies.

The present intervention study includes subjects who were still working at least half time (e.g. sick leave no more than $50 \%$ of normal full time), and most of them were in fact working regular fulltime. In this regard, the intervention could be characterized as an early—or preventive treatment. To what degree the same type of intervention focus on the recovery aspects of stress problems would be comparably successful also in a sample with more serious clinical problems, for example with subjects already on sick-leave due to burnout or exhaustion problems, is an interesting and important, but of course also a empirical question. Given the importance of deficiencies in recovery processes recently shown in both the etiology of burnout and in the recovery from or rehabilitative process of burnout [64], we do however propose that active recovery enhancing intervention components such as those used in our program could be incorporated in more comprehensive treatment programs for such clinical groups as well.

From a practical and therapeutic point of view, our experience is that the intervention focus and format works satisfactory. The rationale for focusing narrowly on recovery behavior, and the different active intervention components included are generally readily accepted by the participants. Also the group leaders' handling group processes and discussions with this focus is most often rather straightforward. This positive impression of the practical usability fits well with earlier experiences from other areas where comparable intervention formats have been used[33] as well as from the several earlier pilot trials of the present project we have carried out [61].

Our general practical aim regarding the intervention format has been that it should have a simplicity and directness, which could make it easy to disseminate, maintain and make available for different contexts and intervention activities. If the positive results achieved in the present study can be confirmed in future studies with more stringent methodological design, the intervention would thus hopefully increase the opportunities and possibilities to help large groups of people in risk of stress related illness or already on sick leave due to problems of this kind-people that until now, unfortunately, have often been left without empirically based, specific, active prevention treatment or rehabilitation efforts.

\section{Acknowledgements}

This work was supported in part by grant \#2010-0654 from FORTE: Swedish Research Council for Health Working Life and Welfare. The contributions of Mary Lam Eriksson at the City Council of Ostersund in organizing the recruitment of participants, and of Åsa Eklund, Magdalena Moström and Catarina Olsson at the Swedish Winter Sports Research Center (NVC), Mid Sweden University for planning and leading the complementary part of the intervention at Swedish Winter Sports \& Research Center (NVC) are greatly appreciated. We are grateful for and impressed by the contributions by the "student therapists" Sofia Djusberg, Johan Ekström, Johan Landin, and Amanda Simonsson. We would like to express our gratitude to the participants, who gave priority, and gave up valuable time to take part in the study.

\section{References}

[1] The Work Environment Authority (2004) Work-Related Disorders 2004. Swedish Work Environment Authority, Publication Services. 
[2] Selye, H. (1950) Stress: The Physiology and Pathology of Exposure to Stress. ACTA Publications, Montreal.

[3] McEwen, B.S. (1998) Stress, Adaptation, and Disease. Allostasis and Allostatic Load. Annals of the New York Academy of Sciences, 840, 33-44.

[4] Eriksen, H.R., Murison, R., Pensgaard, A.M. and Ursin, H. (2005) Cognitive Activation Theory of Stress (CATS): From Fish Brains to the Olympics. Psychoneuroendocrinology, 30, 933-938.

[5] Ursin, H. and Eriksen, H.R. (2004) The Cognitive Activation Theory of Stress. Psychoneuroendocrinology, 29, 567592.

[6] Linden, W., Earle, T.L., Gerin, W. and Christenfeld, N. (1997) Physiological Stress Reactivity and Recovery: Conceptual Siblings Separated at Birth? Journal of Psychosomatic Research, 42, 117-135.

[7] Schwartz, A.R., Gerin, W., Davidson, K.W., Pickering, T.G., Brosschot, J.F., Thayer, J.F., et al. (2003) Toward a Causal Model of Cardiovascular Responses to Stress and the Development of Cardiovascular Disease. Psychosomatic Medicine, 65, 22-35.

[8] Pieper, S. and Brosschot, J.F. (2005) Prolonged Stress-Related Cardiovascular Activation: Is There Any? Annals of Behavioral Medicine, 30, 91-103.

[9] Brosschot, J.F., Gerin, W. and Thayer, J.F. (2006) The Perseverative Cognition Hypothesis: A Review of Worry, Prolonged Stress-Related Physiological Activation, and Health. Journal of Psychosomatic Research, 60, 113-124.

[10] Brosschot, J.F., Pieper, S. and Thayer, J.F. (2005) Expanding Stress Theory: Prolonged Activation and Perseverative Cognition. Psychoneuroendocrinology, 30, 1043-1049.

[11] Brosschot, J.F., Verkuil, B. and Thayer, J.F. (2010) Conscious and Unconscious Perseverative Cognition: Is a Large Part of Prolonged Physiological Activity Due to Unconscious Stress? Journal of Psychosomatic Research, 69, 407-416. http://dx.doi.org/10.1016/j.jpsychores.2010.02.002

[12] Larsen, B.A. and Christenfeld, N.J.S. (2011) Cognitive Distancing, Cognitive Restructuring, and Cardiovascular Recovery from Stress. Biological Psychology, 86, 143-148. http://dx.doi.org/10.1016/j.biopsycho.2010.02.011

[13] Geurts, S.A. and Sonnentag, S. (2006) Recovery as an Explanatory Mechanism in the Relation between Acute Stress Reactions and Chronic Health Impairment. Scandinavian Journal of Work, Environment \& Health, 32, 482-492. http://dx.doi.org/10.5271/sjweh.1053

[14] Zijlstra, F.R.H. and Sonnentag, S. (2006) After Work Is Done: Psychological Perspectives on Recovery from Work. European Journal of Work and Organizational Psychology, 15, 129-138. http://dx.doi.org/10.1080/13594320500513855

[15] Glynn, L.M., Christenfeld, N. and Gerin, W. (2002) The Role of Rumination in Recovery from Reactivity: Cardiovascular Consequences of Emotional States. Psychosomatic Medicine, 64, 714-726.

[16] Gerin, W., Davidson, K.W., Christenfeld, N.J., Goyal, T. and Schwartz, J.E. (2006) The Role of Angry Rumination and Distraction in Blood Pressure Recovery from Emotional Arousal. Psychosomatic Medicine, 68, 64-72. http://dx.doi.org/10.1097/01.psy.0000195747.12404.aa

[17] Chafin, S., Christenfeld, N. and Gerin, W. (2008) Improving Cardiovascular Recovery from Stress with Brief Poststress Exercise. Health Psychology, 27, 64-72. http://dx.doi.org/10.1037/0278-6133.27.1(Suppl.).S64

[18] Chafin, S., Roy, M., Gerin, W. and Christenfeld, N. (2004) Music Can Facilitate Blood Pressure Recovery from Stress. British Journal of Health Psychology, 9, 393-403. http://dx.doi.org/10.1348/1359107041557020

[19] Christenfeld, N. and Gerin, W. (2000) Social Support and Cardiovascular Reactivity. Biomedicine \& Pharmacotherapy, 54, 251-257. http://dx.doi.org/10.1016/S0753-3322(00)80067-0

[20] Tucker, P., Dahlgren, A., Akerstedt, T. and Waterhouse, J. (2008) The Impact of Free-Time Activities on Sleep, Recovery and Well-Being. Applied Ergonomics, 39, 653-662. http://dx.doi.org/10.1016/j.apergo.2007.12.002

[21] Akerstedt, T. (2004) Sleep as Recovery after Stress. Lakartidningen, 101, 1501-1505.

[22] Soderstrom, M., Jeding, K., Ekstedt, M., Perski, A. and Akerstedt, T. (2012) Insufficient Sleep Predicts Clinical Burnout. Journal of Occupational Health Psychology, 17, 175-183. http://dx.doi.org/10.1037/a0027518

[23] Ekstedt, M., Soderstrom, M., Akerstedt, T., Nilsson, J., Sondergaard, H.P. and Aleksander, P. (2006) Disturbed Sleep and Fatigue in Occupational Burnout. Scandinavian Journal of Work, Environment \& Health, 32, 121-131. http://dx.doi.org/10.5271/sjweh.987

[24] Ekstedt, M., Soderstrom, M. and Akerstedt, T. (2009) Sleep Physiology in Recovery from Burnout. Biological Psychology, 82, 267-273. http://dx.doi.org/10.1016/j.biopsycho.2009.08.006

[25] Akerstedt, T., Kecklund, G. and Axelsson, J. (2007) Impaired Sleep after Bedtime Stress and Worries. Biological Psychology, 76, 170-173. http://dx.doi.org/10.1016/j.biopsycho.2007.07.010

[26] Brosschot, J.F., Van Dijk, E. and Thayer, J.F. (2007) Daily Worry Is Related to Low Heart Rate Variability during 
Waking and the Subsequent Nocturnal Sleep Period. International Journal of Psychophysiology, 63, 39-47. http://dx.doi.org/10.1016/j.ijpsycho.2006.07.016

[27] Amstad, F.T. and Semmer, N.K. (2009) Recovery and the Work-Family Interface. In: Sonnentag, S., Perrewé, P.L. and Ganster, D.C., Eds., Current Perspectives on Job-Stress Recovery, Emarald Group Publiching Ltd., Bingley, 125-166.

[28] Statistics Sweden (2014) Women and Men in Sweden: Facts and Figures 2014.

[29] Frankenhaeuser, M., Lundberg, U., Fredrikson, M., Melin, B., Tuomisto, M., Myrsten, A.L., et al. (1989) Stress on and off the Job as Related to Sex and Occupational Status in White-Collar Workers. Journal of Organizational Behavior, 10, 321-346. http://dx.doi.org/10.1002/job.4030100404

[30] Lundberg, U. and Frankenhaeuser, M. (1999) Stress and Workload of Men and Women in High-Ranking Positions. Journal of Occupational Health Psychology, 4, 142-151. http://dx.doi.org/10.1037/1076-8998.4.2.142

[31] Lundberg, U. and Cooper, C.L. (2010) The Science of Occupational Health: Stress, Psychobiology, and the New World of Work. John Wiley \& Sons, Hoboken. http://dx.doi.org/10.1002/9781444391121

[32] Soares, J.J.F., Grossi, G. and Sundin, O. (2007) Burnout among Women: Associations with Demographic/Socio-Economic, Work, Life-Style and Health Factors. Archives of Women's Mental Health, 10, 61-71.

[33] Lisspers, J. and Almén, N. (2009) Föllingemodellen-En KBT-inriktad och internatsbaserad interventionsmodell för beteendeförändring vid livsstils-och stressrelaterad ohälsa. Folksam/Folksam Hälsa, Stockholm.

[34] Laaksonen, M.S., Ainegren, M. and Lisspers, J. (2011) Evidence of Improved Shooting Precision in Biathlon after 10 Weeks of Combined Relaxation and Specific Shooting Training. Cognitive Behaviour Therapy, 40, 237-250. http://dx.doi.org/10.1080/16506073.2011.616217

[35] Burell, G. (1996) Group Psychotherapy in Project New Life: Treatment of Coronary Prone Behavior in Coronary Bypass Graft Surgery Patients. In: Allan, R. and Scheidt, S., Eds., Heart \& Mind: The Practice of Cardiac Psychology, American Psychological Association, Washington DC, 291-310.

[36] Claesson, M., Burell, G., Birgander, L.S., Lindahl, B. and Asplund, K. (2003) Psychosocial Distress and Impaired Quality of Life-Targets Neglected in the Secondary Prevention in Women with Ischaemic Heart Disease. Journal of Cardiovascular Risk, 10, 258-266.

[37] Leineweber, C., Wege, N., Westerlund, H., Theorell, T., Wahrendorf, M. and Siegrist, J. (2010) How Valid Is a Short Measure of Effort-Reward Imbalance at Work? A Replication Study from Sweden. Occupational and Environmental Medicine, 67, 526-531. http://dx.doi.org/10.1136/oem.2009.050930

[38] Siegrist, J., Starke, D., Chandola, T., Godin, I., Marmot, M., Niedhammer, I., et al. (2004) The Measurement of EffortReward Imbalance at Work: European Comparisons. Social Science \& Medicine, 58, 1483-1499. http://dx.doi.org/10.1016/S0277-9536(03)00351-4

[39] Siegrist, J., Wege, N., Pühlhofer, F. and Wahrendorf, M. (2008) A Short Generic Measure of Work Stress in the Era of Globalization: Effort-Reward Imbalance. International Archives of Occupational and Environmental Health, 82, 10051013. http://dx.doi.org/10.1007/s00420-008-0384-3

[40] Berle, D., Starcevic, V., Moses, K., Hannan, A., Milicevic, D. and Sammut, P. (2011) Preliminary Validation of an Ultra-Brief Version of the Penn State Worry Questionnaire. Clinical Psychology \& Psychotherapy, 18, 339-346. http://dx.doi.org/10.1002/cpp.724

[41] Meyer, T.J., Miller, M.L., Metzger, R.L. and Borkovec, T.D. (1990) Development and Validation of the Penn State Worry Questionnaire. Behaviour Research and Therapy, 28, 487-495. http://dx.doi.org/10.1016/0005-7967(90)90135-6

[42] Cohen, S., Kamarck, T. and Mermelstein, R. (1983) A Global Measure of Perceived Stress. Journal of Health and Social Behavior, 24, 385-396. http://dx.doi.org/10.2307/2136404

[43] Gustafsson, K., Lindfors, P., Aronsson, G. and Lundberg, U. (2006) Validation of Unwinding and Recuperation Questions: Correlations between Salivary Cortisol and Subjective Ratings. Arbetslivsinstitutet, Arbete och Hälsa, Stockholm.

[44] Von Thiele, U., Lindfors, P. and Lundberg, U. (2006) Self-Rated Recovery from Work Stress and Allostatic Load in Women. Journal of Psychosomatic Research, 61, 237-242. http://dx.doi.org/10.1016/j.jpsychores.2006.01.015

[45] Melamed, S., Kushnir, T. and Shirom, A. (1992) Burnout and Risk Factors for Cardiovascular Diseases. Behavioral Medicine, 18, 53-60. http://dx.doi.org/10.1080/08964289.1992.9935172

[46] Shirom, A., Westman, M., Shamai, O. and Carel, R.S. (1997) Effects of Work Overload and Burnout on Cholesterol and Triglycerides Levels: The Moderating Effects of Emotional Reactivity among Male and Female Employees. Journal of Occupational Health Psychology, 2, 275-288. http://dx.doi.org/10.1037/1076-8998.2.4.275

[47] Melamed, S., Ugarten, U., Shirom, A., Kahana, L., Lerman, Y. and Froom, P. (1999) Chronic Burnout, Somatic Arousal and Elevated Salivary Cortisol Levels. Journal of Psychosomatic Research, 46, 591-598. 
http://dx.doi.org/10.1016/S0022-3999(99)00007-0

[48] Zigmond, A.S. and Snaith, R.P. (1983) The Hospital Anxiety and Depression Scale. Acta Psychiatrica Scandinavica, 67, 361-370. http://dx.doi.org/10.1111/j.1600-0447.1983.tb09716.x

[49] Lisspers, J., Nygren, A. and Soderman, E. (1997) Hospital Anxiety and Depression Scale (HAD): Some Psychometric Data for a Swedish Sample. Acta Psychiatrica Scandinavica, 96, 281-286. http://dx.doi.org/10.1111/j.1600-0447.1997.tb10164.x

[50] Kecklund, G. and Akerstedt, T. (1992) The Psychometric Properties of the Karolinska Sleep Questionnaire. Journal of Sleep Research, Suppl. 1, 113.

[51] Leineweber, C., Kecklund, G., Akerstedt, T., Janszky, I. and Orth-Gomér, K. (2003) Snoring and the Metabolic Syndrome in Women. Sleep Medicine, 4, 531-536. http://dx.doi.org/10.1016/S1389-9457(03)00160-6

[52] Cohen, J. (1992) A Power Primer. Psychological Bulletin, 112, 155-159. http://dx.doi.org/10.1037/0033-2909.112.1.155

[53] Feingold, A. (2009) Effect Sizes for Growth-Modeling Analysis for Controlled Clinical Trials in the Same Metric as for Classical Analysis. Psychological Methods, 14, 43-53. http://dx.doi.org/10.1037/a0014699

[54] Larsen, B.A. and Christenfeld, N.J.S. (2009) Cardiovascular Disease and Psychiatric Comorbidity: The Potential Role of Perseverative Cognition. Cardiovascular Psychiatry and Neurology, 2009, 1-8. http://dx.doi.org/10.1155/2009/791017

[55] Sundin, L., Hochwälder, J. and Lisspers, J. (2011) A Longitudinal Examination of Generic and Occupational Specific Job Demands, and Work-Related Social Support Associated with Burnout among Nurses in Sweden. Work, 38, 389400.

[56] Stavosky, J.M. and Borkovec, T.D. (1987) The Phenomenon of Worry: Theory, Research, Treatment and Its Implications for Women. Women \& Therapy, 6, 77-95. http://dx.doi.org/10.1300/J015V06N03_07

[57] McCann, S.J.H., Stewin, L.L. and Short, R. (1991) Sex Differences, Social Desirability, Masculinity, and the Tendency to Worry. Journal of Genetic Psychology, 152, 295-301. http://dx.doi.org/10.1080/00221325.1991.9914687

[58] Lewinsohn, P.M., Gotlib, I.H., Lewinsohn, M., Seeley, J.R. and Allen, N.B. (1998) Gender Differences in Anxiety Disorders and Anxiety Symptoms in Adolescents. Journal of Abnormal Psychology, 107, 109-117. http://dx.doi.org/10.1037/0021-843X.107.1.109

[59] Robichaud, M., Dugas, M.J. and Conway, M. (2003) Gender Differences in Worry and Associated Cognitive-Behavioral Variables. Journal of Anxiety Disorders, 17, 501-516. http://dx.doi.org/10.1016/S0887-6185(02)00237-2

[60] Almeida, D.M. and Kessler, R.C. (1998) Everyday Stressors and Gender Differences in Daily Distress. Journal of Personality and Social Psychology, 75, 670-680. http://dx.doi.org/10.1037/0022-3514.75.3.670

[61] Almen, N., Sundin, O. and Lisspers, J. (2012) Behavioral Intervention for "Recovery Management”-A Pilot Study with Single-Subject Experimental Design. Department of Psychology, Mid Sweden University Campus Ostersund, Sweden (Submitted).

[62] Barlow, D.H., Nock, M.K. and Hersen, M. (2008) Single Case Experimental Designs: Strategies for Studying Behavior Change. 3rd Edition, Allyn \& Bacon, New York.

[63] Hayes, S.C., Barlow, D.H. and Nelson-Gray, R.O. (1999) The Scientist Practitioner: Research and Accountability in the Age of Managed Care. 2nd Edition, Allyn \& Bacon, Needham Heights.

[64] Soderstrom, M. (2012) Burnout-A Matter of Impaired Recovery? Doctoral Dissertation, Karolinska Institute, Stockholm. 
Scientific Research Publishing (SCIRP) is one of the largest Open Access journal publishers. It is currently publishing more than 200 open access, online, peer-reviewed journals covering a wide range of academic disciplines. SCIRP serves the worldwide academic communities and contributes to the progress and application of science with its publication.

Other selected journals from SCIRP are listed as below. Submit your manuscript to us via either submit@scirp.org or Online Submission Portal.
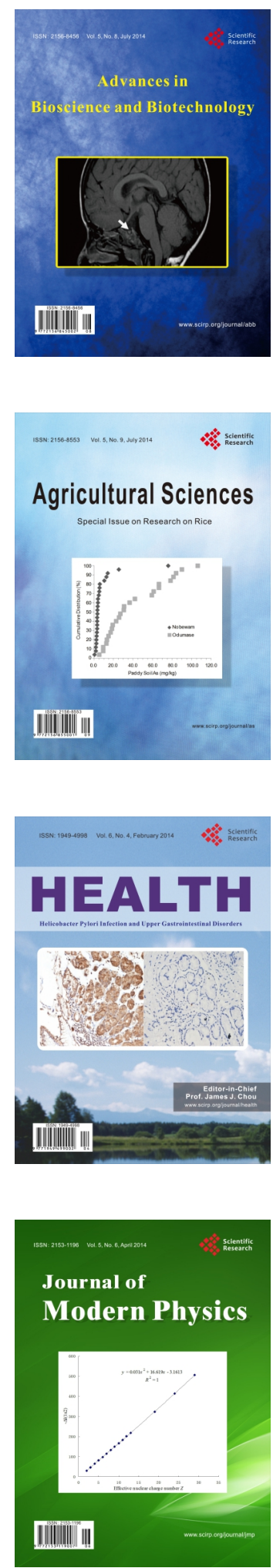
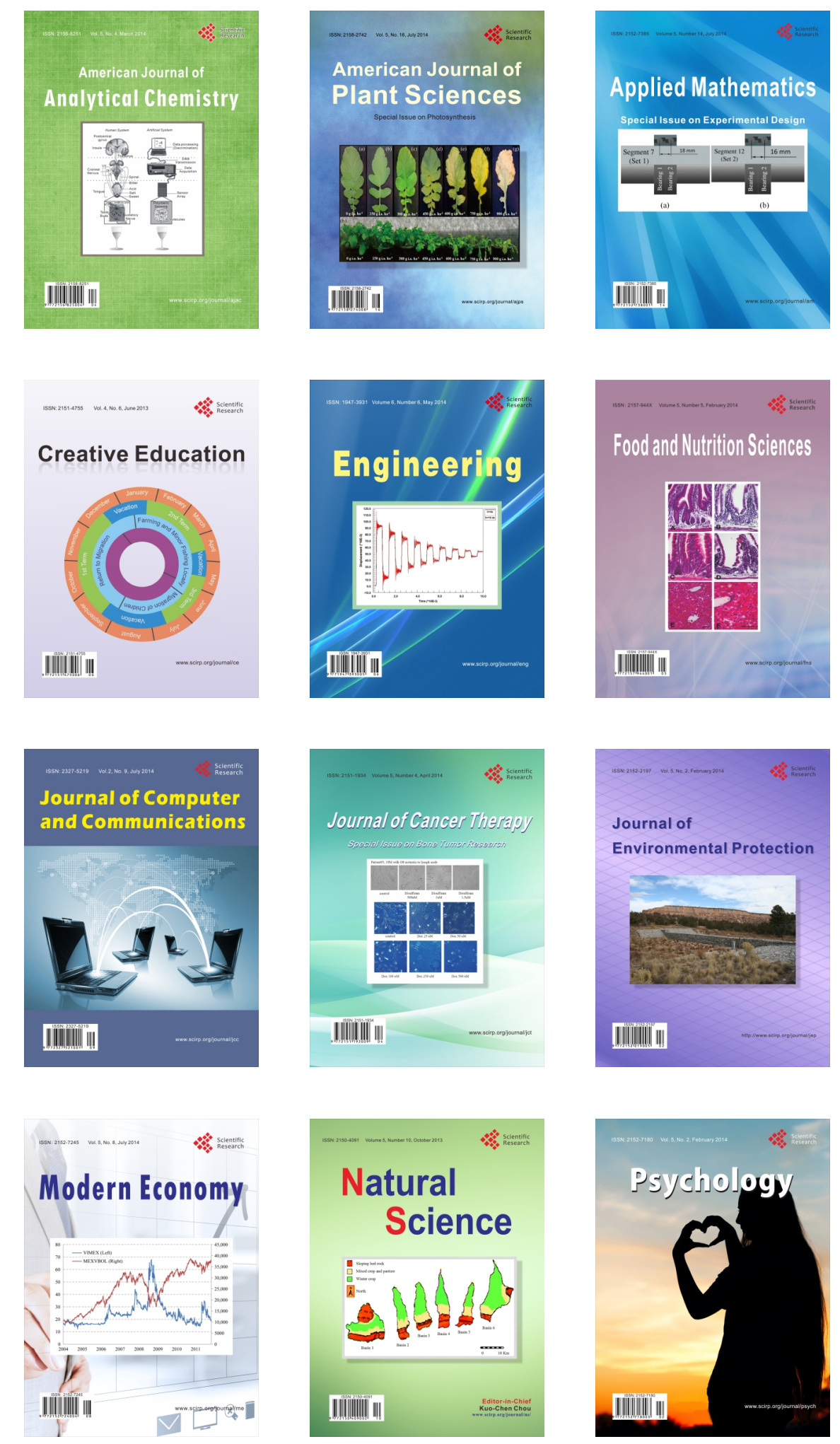